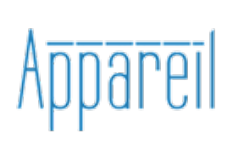

Appareil

$2 \mid 2008$

Autour de Simondon

\title{
Technique et humanisme. Günther Anders et Gilbert Simondon
}

\section{Giovanni Carrozzini}

\section{(2) OpenEdition}

1 Journals

\section{Édition électronique}

URL : http://journals.openedition.org/appareil/577

DOI : 10.4000/appareil.577

ISSN : 2101-0714

Éditeur

MSH Paris Nord

Référence électronique

Giovanni Carrozzini, «Technique et humanisme. Günther Anders et Gilbert Simondon », Appareil [En ligne], 2 | 2008, mis en ligne le 05 septembre 2008, consulté le 30 juillet 2020. URL : http:// journals.openedition.org/appareil/577; DOI : https://doi.org/10.4000/appareil.577

Ce document a été généré automatiquement le 30 juillet 2020.

\section{(c) (i) (3)}

Appareil est mis à disposition selon les termes de la Licence Creative Commons Attribution - Pas d'Utilisation Commerciale - Pas de Modification 4.0 International. 


\title{
Technique et humanisme. Günther Anders et Gilbert Simondon
}

\author{
Giovanni Carrozzini
}

1 «Ducunt fata volentem, nolentem trahunt ». C'est avec ces mots de Sénèque, lecteur de Cléante, que se termine une des plus fameuses apocalypses $d u x^{e}$ siècle. C'est Oswald Spengler qui les emploie, en 1922, à la fin de son Déclin de l'Occident, causé par une naturelle consomption de la Kultur occidentale par la diffusion d'une Zivilisation qui possède, à son tour, un visage précis : la Technique. Une technique en majuscules, qui trace les voies et qui se confond avec le destin de l'Occident. On pourrait donc se demander avec Lénine : « que faire? »C'est toujours Spengler qui nous répond, dans un bref texte, paru en 1931, intitulé L'homme et la technique: l'auteur imagine une seule solution à la question posée par le contexte historique dans lequel il se trouvait à penser: attendre la ruine sans laisser sa propre place. C'est la Technique, selon Spengler, qui nous oblige à cette condamnation à la passivité, et c'est le cours de l'histoire qui nous pose un terminus ad quem. Pendant l'époque de la Technique, l'homme doit donc accepter l'idée stoïcienne d'Epictète : « Accepte-toi. Renonce-toi ».

2 Les périodes de crise - et la période pendant laquelle Spengler pensait et rédigeait son livre était sans doute une de ces périodes - ont toujours divisé les hommes en deux groupes : ceux qui cherchent à comprendre et ceux qui se désespèrent. On peut, donc, penser l'histoire comme un système sujet à des épisodes catastrophiques; René Thom l'a dit parfaitement : après un épisode catastrophique les dynamiques et les équilibres $\mathrm{du}$ système se dispersent, ou bien ils changent d'une telle façon qu'on ne peut plus retrouver dans le présent ce qu'il y avait dans le passé. Mais aussi l'histoire possède ses lois et les lois de la science nouvelle ce sont les lois de l'homme, qui représente, à son tour, l'objet privilégié de l'histoire. C'est pour cette raison que je crois pouvoir retrouver des répétitions dans le cours « chaotique » de l'histoire humaine : ces lois sont les catastrophes, et les catastrophes sont des crises qui ont une période qui les prépare et une période pendant laquelle on contemple ses conséquences. Le $\mathrm{xx}^{\mathrm{e}}$ siècle pourrait être pensé comme une phase dans laquelle on a vu se dérouler trente ans de guerre, et 
un moment - de presque quarante années - qui payait les dommages de ces trente ans " de fer, de sang et d'acier ", comme disait Prévert.

3 Si j'ai commencé en vous exposant les thèses conclusives de Spengler, c'est surtout pour vous introduire à une analogie : celle qu'on pourrait construire entre les vingt ans qui précèdent la Grande Guerre et ceux qui séparent les deux guerres mondiales, mais c'est aussi pour vous illustrer la conscience des intellectuels devant les catastrophes et leur réflexion à cet égard, une réflexion qui, à cause de sa structure intrinsèque même (réfléchir signifie penser après), est semblable à l'animal que Hegel imaginait pour la philosophie : la chouette qui vole à la fin du jour, après que toutes les actions des hommes ont trouvé leur conclusion.

4 On a déjà dit que les catastrophes influencent les opinions des hommes, et plus généralement elles vont changer leur approches du réel : les intellectuels, pendant ces moments, deviennent des véritables papiers de tournesol pour saisir la mentalité qui va se diffuser ou bien qui s'est déjà diffusée dans une "culture ». Spengler était sans doute l'exemple le plus efficace pour comprendre l'Allemagne de Weimar, qui voyait approcher sa fin. C'est le désespoir qui avance : toutefois, toujours selon Spengler, on doit rester à sa propre place, même si c'est l'incipit tragoedia. Tout au contraire, il y a aussi des cas historiques dans lesquels la conscience et le désespoir se partagent l'âme du même homme et on les voit surgir dans des moments différents de son existence. En même temps, ce qui reste de cet homme, c'est sans doute son dernier mot, sa dernière façon de penser le réel, et même si conscience et désespoir se sont défiés en combat singulier dans son esprit, il y a toujours un vainqueur qui triomphe et qui peint le portrait futur de cet homme.

5 J'ai beaucoup réfléchi sur la modalité la plus efficace de vous présenter Günther Anders, ou bien, mon Günther Anders, c'est-à-dire la façon dont - plus ou moins volontairement - j'ai appris à le lire et à l'entendre. Anders a été un exceptionnel modulateur de la crise allemande pendant le nazisme, un miroir pour voir et comprendre la condition des hébreux pendant le délire hitlérien. C'est pour cela qu'on pourrait donc le penser - comme a déjà fait Pierpaolo Portinaro - comme le témoin de l'apocalypse, en y voyant une approche pas du tout différente de celle adoptée par Spengler pendant les années de la Grande Guerre. Toutefois, je ne suis pas sûr que celle-ci serait la fresque la meilleure qu'on pourrait donner de la complexité qui est soumise à la philosophie occasionnelle d'Anders, selon l'expression qu'il avait choisi pour décrire sa façon de penser le présent. Cette image d'Anders se limite à nous donner seulement les lignes finales, les résultats théorétiques d'Anders, mais elle tait tout ce qui a préparé ce donné. On sait bien qu'au contraire pour comprendre une pensée il est mieux de parcourir ses chemins, ses ruptures et ses "longs détours ", sa chair et son sang, puisque la pensée est faite de la chair même de son artisan, et moi, je n'ai jamais cru au ciel platonicien, habité par des idées éternelles, qui préexistent aux hommes qui les pensent. Anders a été donc le sismographe d'une crise, la conscience de cette crise, même si, à la fin de son parcours intellectuel, il n'a pas réussi à donner une véritable solution philosophique à cette crise et il a donc préféré l'abandon, la seule pars destruens, quelque fois stérile et autoréférentielle, au statu quo, en choisissant la passivité plutôt que l'action de la raison qui, si on y croit, possède toujours des moyens pour sortir des instants dans lesquels toutes les vaches semblent noires, pour le dire avec Hegel. Mais j'ai dit qu'il a été aussi une conscience du siècle : cela veut dire qu'il a aussi essayé de comprendre avant de critiquer : il y a, en effet, une phase précise dans laquelle Anders démontre ce besoin 
illuministe, ce rationalisme critique à l'égard de sa période. Ce moment correspond aux années qui ont précédé 1933, une période durant laquelle l'Allemagne avait eu la possibilité de comprendre et connaître son futur Führer à travers le Mein Kampf, publié en 1926, et qui était le plus clair programme politique du futur nazisme. Anders fait partie d'une minorité objective : celle de ceux qui vont lire ce programme et qui en soupèsent le péril. C'est toujours Anders qui le dénonce et qui accuse les Allemands, et plus encore les intellectuels de son époque de n'avoir pas fait une des opérations les plus naturelles à faire avant d'agir : connaitre. Anders avait lu ce livre, Anders avait analysé les conséquences d'une victoire politique du national-socialisme, Anders avait déjà imaginé et écrit les ruines du futur: toutefois, il était orphelin d'une qualité nécessaire aux penseurs de toutes époques: il n'avait pas (encore) une véritable autonomie de pensée pour le dénoncer et surtout il ne trouvait pas des maîtres capables de partager ses préoccupations. Cette histoire, que je vais vous exposer, est vraiment bizarre : élève intelligent, journaliste habile et aigu, Anders restait encore séduit par " les yeux » et " les mots » d'un homme qui tout au contraire n'avait aucune estime de lui, un "monstre sacré de la pensée philosophique " qui était conscient d'avoir baptisé une époque avec son nom et d'avoir ouvert une nouvelle façon de penser l'Être : ce personnage, vous l'avez déjà compris, c'est Heidegger. Dans une lettre de 1925 à Hannah Arendt, qui épousera plus tard Anders tout en continuant son rapport avec Heidegger, le philosophe de Sein und Zeit décrit Stern - Anders, comme vous le savez déjà, c'était seulement un pseudonyme - comme un homme pénible, qui cherchait des conseils tout en démontrant n'avoir pas une véritable maturité philosophique. Tout au contraire, Anders se disait en ce temps disciple d'Heidegger, charmé par son œuvre de 1927, prêt à excuser les humeurs et les velléités académiques de son maître. Et la fascination qu'Heidegger avait exercé sur Anders se perpétue aussi au lendemain de la guerre, de son implication avec le nazisme, qu'Heidegger n'a jamais reniée, comme on peut le constater en lisant le terrible entretien avec les correspondants de la revue Der Spiegel du 1976. Même si Anders démontrera plus tard avoir compris les limites de la pensée heideggérienne, qu'il va dénoncer dans plusieurs fragments dédiés au philosophe, il reste toujours un certain héritage heideggérien dans sa façon de philosopher: ce n'est pas seulement son manque de confiance dans la raison humaine qui le rapproche de Heidegger, c'est-à-dire le fait que les deux penseurs croyaient à la raison des illuministes comme à une ratio de la démesure, semblable à une hybris plutôt qu'à une lumière. C'est aussi et surtout le fait que tous les deux exigeaient un nouveau sujet pour l'histoire qui ne pouvait plus être l'homme : c'est leur commun désespoir à l'égard de chaque philosophie humaniste qui crée le trait d'union entre leurs réflexions. Heidegger et Anders voient dans l'homme un corps cadavérique, une ruine plutôt qu'un protagoniste: c'est toujours quelque chose d'autre qui conduit l'histoire et qui en même temps doit être conduit. Toutefois, dans la mesure où il faudrait le conduire, c'est toujours comme gardiens et pas comme chef d'orchestre qu'il faudra le faire. L'histoire, selon Heidegger et Anders, n'est plus le verum est factum de Vico, parce que le verum ne coïncide plus avec le factum, et ce n'est plus l'homme qui bâtit son futur, qui organise ses fêtes et ses funérailles.

6 Tandis que Sartre, en 1946, déclarait que l'existentialisme ne peut qu'être un humanisme, c'est-à-dire un discours centré autour du "personnage-homme», avec toutes ses limites, ses faiblesses, mais surtout avec ses capacités d'arraisonner le cours de sa propre existence, toujours en 1946, Heidegger lui répondait indirectement avec une Brief über den "Humanismus" (adressée à Jean Beaufret), dans laquelle il allait 
dénoncer son manque de confiance à l'égard de l'homme. "Précisément - il affirme nous sommes sur un plan où il y a principalement l'Être ». L'usage du mot principalement ne signifie pas qu'il croit dans une coexistence de l'homme et de l'Être posés sur le même plan, tout au contraire, il croit que sur la scène du monde on observe un seul acteur et quelqu'un qui lui sert de faire-valoir : celui-ci c'est l'homme, qui doit donc abandonner sa propre activité pour lui préférer une sorte de passivité de la pensée. Même si Heidegger ne croyait pas que l'histoire était arrivée à sa fin, il ne voyait pas dans l'époque présente une progression de faits humains trop humains: au contraire, il y saisissait le triomphe d'une nouvelle philosophie, ou bien, l'occasion pour rétablir une nouvelle façon de penser la pensée philosophique: cette nouvelle façon, il l'appelait Andenken, en employant un terme cher au poète Hölderlin qui, comme vous le savez, avait inspiré sa deuxième phase philosophique, celle que nous connaissons comme Kehre. Les années $40 \mathrm{du} \mathrm{xx}^{\mathrm{e}}$ siècle n'étaient pas encore la période dans laquelle Anders avait produit son chef d'œuvre, qui va paraître en 1956 : au même moment, il allait préparer son détachement par rapport à la philosophie de son maître, qu'il critiquait en employant des métaphores, des argumentations stylistiques plutôt que purement philosophiques. Heidegger restait pour lui l'auteur d'une œuvre qui avait changé le cours de l'histoire de la philosophie et même au lendemain de son exil, sa haine se confondait encore avec son estime intellectuelle. En 1953, Heidegger présente au public une conférence dédiée à un thème qui deviendra un de ses nouveaux philosophema et qui était déjà apparu, mais seulement comme corollaire, dans Sein und Zeit : je parle de la technique et donc de la conférence intitulée Die Frage nach der Technik, dans laquelle il présente une transformation radicale des fondements de la technique moderne par rapport à la technique traditionnelle, conçue, à la manière des Grecs, comme techné, donc comme expression d'un dévoilement de l'Être de sa propre maison. À cet égard, concédez-moi de vous rappeler l'image du bûcheron qui connaissait les arbres de la forêt versus les machines qui sont employées aujourd'hui pour la déforestation. Mais Heidegger, en suivant encore une fois Hölderlin, n'est pas un prophète du déluge. Il réussit à voir une expression de salut futur pour l'homme par rapport à la technique moderne : ce n'est pas la philosophie, pas du tout non plus la science, mère dénaturée de la technique moderne, mais bien plutôt l'art qui pourrait rétablir ce lien entre homme et technique, même s'il ne décrit pas les temps, les conditions et plus encore les raisons intimes pour lesquelles l'art peut faire le miracle. Je ne sais pas s'il faut le croire quand, pendant l'entretien de 1976, que j'ai déjà cité, il arrive à affirmer que c'était le nazisme qui avait compris cette nouvelle sorte de coexistence entre homme et technique : ce que je sais est que Heidegger voyait la place de la philosophie volée par la Cybernétique, et l'homme toujours semblable à l'essence de ce wartete Leute qui devait entendre et conduire l'Être, un peuple qui, à son tour, possédait une identité précise : le peuple de Goethe, d'Hölderlin et de Wagner, c'est-à-dire le peuple de la bête blonde, comme l'avait défini Nietzsche, le peuple allemand, le seul capable d'établir cette nouvelle alliance avec l'Être, méconnu par l'histoire de la métaphysique traditionnelle, de Platon à Nietzsche.

7 Trois années plus tard, en 1956, l'éditeur Verlag publiait le premier volume de l'œuvre d'Anders à laquelle son nom restera lié : on parle de Die Antiquietheit des Menchen, œuvre qui ne présente pas encore le style qui caractérisera le philosophe, c'est-à-dire l'écriture occasionnelle qu'il choisira pour présenter son deuxième volume, paru en 1981, mais qui contient des articles rédigés de 1956 à 1980. Avant tout, je crois qu'il sera nécessaire de vous illustrer une première connexion, encore une fois d'ordre 
strictement stylistique, entre Anders et son maître : après 1927, comme vous le savez bien, Heidegger choisit la forme de l'article ou bien de la conférence pour véhiculer ses réflexions : l'esprit de système qu'il avait incarné avec Sein und Zeit l'abandonne. L'emploi du terme abandon n'est pas casuel: la Kehre dont il parle n'est pas exactement un changement de sa pensée, mais plutôt un bouleversement dans l'Être. C'est pour cela qu'on ne peut pas utiliser le même style pour en parler : il nécessite un nouveau langage et même une diffusion différente de ce qu'on va affirmer à son égard. Anders n'a pas choisi le style du fragment pour les raisons qui avaient conduit son maître à le faire : c'est plutôt une exigence professionnelle qui l'anime. Mais ce qui reste c'est toujours un certain parallélisme entre la façon philosophique d'Heidegger et celle de son ancien élève. Un seul éclaircissement: Anders avait critiqué l'emploi du nouveau langage heideggérien, qu'il voyait comme l'expression la plus complète de son académisme, toutefois la structure et, comme on va voir, l'humus de leurs recherches restent communs.

Comme on l'a déjà dit, Heidegger comme Anders voyaient la place de l'homme remplacée par une nouvelle entité, qui possède des confins qui ne sont pas clairs et dont les propriétés sembleraient être celles d'un malin génie cartesien plutôt que celles d'un produit authentiquement humain qui peut, toujours à cause de l'homme et de son incapacité à la contrôler, s'opposer à son développement. Heidegger disait de la Technique qu'elle était la nouvelle phase de l'Être méconnu par la métaphysique: la technique moderne c'est le Ge-stell, une puissance qui va faire de l'homme sa marionnette, pour le dire cette fois avec Platon, et qui va le mesurer et le calculer comme elle mesure et calcule, par exemple, les forêts, les champs, les ciels etc.

En 1978, dans un article qui va paraître dans le second tome de l'œuvre d'Anders qu'on a déjà citée, l'Auteur se livre à une analyse philosophique du cours de l'histoire : le titre de son écrit est L'histoire. La technique comme sujet de l'histoire. Si Heidegger prophétisait un changement, Anders déclare la fin de l'histoire. On connaît son bref entretien intitulé Si je suis désespéré, qu'est-ce que ça peut bien faire?, dans lequel il ne fait pas mystère de sa profession de foi : le désespoir. La conscience des années 20 et 30, sa rage contre le nazisme est devenue un souvenir lointain, trop lointain pour interagir avec sa nouvelle posture philosophique. Mais voilà : ce n'est pas le nazisme qui semblerait lui avoir volé la conscience de l'action, la nécessité d'être toujours responsable par rapport au monde. Ce n'est pas ça : comme il va le déclarer, c'est plutôt cette troisième révolution industrielle qui monte qui représente la cause de son état d'âme. Et cette révolution n'est pas la montée du secteur industriel ou bien la diffusion et l'emploi d'une nouvelle invention techno-scientifique extraordinaire : rien de cela ne correspond à l'esprit qui domine l'époque contemporaine. C'est plutôt l'ère dans laquelle les machines produisent seulement des machines contre l'homme, en lui volant la liberté, l'égalité, la fraternité. Ou bien en lui donnant une liberté, une égalité et une fraternité qui ne sont pas le fruit d'une bataille, d'une victoire : ce sont plutôt la condamnation de l'indistinction, de l'élision de la présence et de la morale : les moyens - voilà ce que c'est la technique pour Anders ont remplacé les buts. La catastrophe kantienne n'a rien à voir avec ce que soutenait Philonenko : c'est la fin de l'homme, la fin de la dernière question que Kant se posait et donc c'est la destruction de toutes pensées pour l'homme, pour citer Dufrenne, parce que ce sont donc les funérailles de l'homme. Si est vrai ce que Marc Bloch affirmait dans son Apologie pour l'histoire, et donc que l'histoire est l'affaire des hommes, la fin de l'homme est donc pour Anders la fin de l'histoire: nous nous trouvons, avec Anders, dans un scénario semblable à celui tracé par Fukuyama, selon lequel l'histoire est terminée, 
mais ce n'est pas, naturellement, à cause de la chute du communisme, comme dans le cas de Fukuyama, qui la condamne; selon Anders, c'est plutôt la pression d'un bouton qui a levé le rideau sur la scène des ruines : selon Anders, ce bouton est celui qui a condamné à la disparition Hiroshima et Nagasaki, dernier épisode de la puissance technique.

Maintenant, je crois qu'il faut nous arrêter un instant et réfléchir : quelle est l'origine de cette image de la technique ? Y-a-t-il un primum movens qui a inauguré cette façon de penser la science et plus encore la technique ? La raison illuministe a célébré plusieurs fois son trépas, toutefois je crois qu'on peut cueillir un terminus a quo de cette démarche : c'est en 1947, date de parution de l'œuvre de Horkheimer et Adorno qui voyaient, dans La dialectique de l'Illuminisme, le germe du pouvoir réifiant de la raison calculatrice, qui faisait de tous hommes des Ulysse liés au pilier d'un navire qui marchait vers l'inouï et l'inimaginable. L'époque de Horkheimer et Adorno c'est l'époque des hommes qui se dévorent, les uns les autres, comme le Conte Ugolin de Dante Alighieri dévore la nuque de l'Archevêque, tout en employant leur différence spécifique, leur res humaine, c'est-à-dire la raison, qui, toujours selon ces Auteurs, s'est traduit dans l'arme du calcul, le royaume de la science antihumaniste et enfin capitaliste. Et Anders? Après son départ pour l'Amérique en 1936, il se sépare de sa femme Hannah et de son cousin, Walter Benjamin, et il va vivre dans la maison d'un autre Auteur de la théorie critique: Herbert Marcuse. L'Amérique est pendant ces années l'« Autre Allemagne », comme on la connaissait à cause de la présence de plusieurs intellectuels hébreux et allemands exilés - plus ou moins volontairement : "À côté de la maison de Marcuse - dit Anders -, dans laquelle j'ai habité pendant mon séjour californien, habitait Brecht. [...] Schönberg résidait, si je ne me trompe pas, à Westwood. Dans un quartier plus élégant il y avait Horkheimer. A Hollywood Döblin. Et Adorno. » Une occasion de croissance et de débat, mais aussi un thiasos d'intellectuels vaincus par l'histoire qui préparaient leur revanche. L'Amérique était la terre du progrès, des lumières électriques et des avions de Roosevelt, l'Amérique c'était la démocratie : mais aussi pour un apatride comme Anders il y a toujours un coin dans lequel on se sent chez soi et cela n'était pas la Californie, mais plutôt la nation qui l'avait obligé à fuir et dans laquelle la technique s'était transformée en fours crématoires et en Zyclon-B d'Hitler. Mais voilà on voit encore un espoir dans cette terre de la radio et de Charlie Chaplin... elle est peut-être le témoignage que la technique pourrait aider l'homme. Non! Ce n'est pas comme-ça : on va le voir le 25 juillet 1945. Deux siècles nous séparent d'un autre juillet : celui-la était la date de naissance de l'Europe contemporaine, libre, responsable et victorieuse, celuici - et Anders en témoigne - c'est le début de la fin : la Technique nous condamne au déluge, et on ne dispose d'aucune arche pour nous sauver. L'homme est fini, l'histoire est finie : ducunt fata volentem, nolentem trahunt. En 1922 comme en 1945 : c'est la cour du Roi Peste de Poe qui triomphe, les projets du Docteur Frankenstein, et les hommes, tous les hommes, vont périr par perte de conscience, perte de chances pour le futur.

11 Et maintenant, je crois qu'il sera nécessaire de nous poser une question, vieille comme l'aube du monde, mais qui toutefois n'a pas encore trouvé une solution et qui, je crois, ne pourra pas la trouver dans le futur. La philosophie possède-t-elle un usage? C'est une question que Bloch s'était posée à l'égard de l'histoire mais, voilà, encore une fois sa mort nous a empêché de connaître la réponse. Les néo-positivistes diraient que celleci est un pseudo-problème, qu'on se trouve dans l'espace du Sinnloss. Mais encore une fois, qui décide du sens des questions? La logique peut-elle décider pour l'homme? Je ne crois pas que Schlick devant son assassin ait pensé à l'absence totale de sens d'une question sur le sens de la vie. Et donc, essayons de trouver - s'il est possible - une 
solution partielle à cette question. Je n'ai jamais cru que le sens de la philosophie soit seulement le sens d'une question: les philosophes, tous les philosophes « dignes de ce nom » - pour le dire avec Bergson - ont toujours posé des questions, c'est vrai, mais ils ont aussi donné des solutions. Nous ne sommes pas obligés de choisir leurs points de vue, plusieurs d'entre eux ont imaginé un homme qui n'existe pas, plus semblable à un dieu qu'a une créature née dans le sang et l'urine, comme disait Augustín d'Hippone. Et pourtant ils ont donné leur solution : l'homme doit chercher la vertu de la mesotes pour Aristote, la contemplation pour Platon, le retour à l'Un et l'abandon de la matière pour Plotin, l'amor intellectualis pour Spinoza, la volonté de puissance pour Nietzsche etc. C'est à cause du fait que dans sa chair on a inscrit un projet qui l'oblige au choix, ou bien, le fait qu'il y inscrit un projet qui l'oblige à sa liberté de choisir. Anders l'avait compris en 1936-1937, à l'époque de sa Pathologie de la liberté, deux textes qu'il disait antérieurs à l'intuition sartrienne, mais voilà en 1956-1981 "tout est abîmé », mis entre parenthèses, remplacé par l'annonce de la septième trombe, la rupture du septième sceau. Mais si la philosophie a un sens, si la philosophie possède un emploi, il est de donner aux hommes une direction et un vers possible, toujours faillibles mais objectifs en tant que solutions, propositions de solution. Anders a manqué sa cible, il est resté regarder la scène, tout en oubliant le conseil illuministe de «se salir les mains avec le monde entier ». Toutes les tragédies, même les plus terribles, même la mort de nos parents, le génocide de notre peuple, si nous restons occuper une place sur cette planète, nous obligent à la responsabilité de comprendre : « pas la mort de ton père disait Averroès - peut justifier ta façon d'agir après vingt ans ».

Il y a un autre problème à résoudre, le problème qui nous introduira dans la philosophie de Simondon : le monde, voilà, ne possède plus de lendemain, le futur est celui du Totale Mobilmachung mais pourquoi faut-il croire que c'est la technique qui nous a donné ce destin ou bien qui est ce destin? La technique reste toujours une des plus dignes expressions de notre être au monde: mais la technique, cela est vrai, traverse une période de méconnaissance qui a paradoxalement commencé avec l'époque de sa technophanie. Aujourd'hui, mais on pourrait dire dès le $\mathrm{xx}^{\mathrm{e}}$ siècle, on voit la technique partout, mais la différence est qu'aujourd'hui on reste seulement à la contempler ou bien à l'employer, on l'aliène de la sphère de la compréhension, on la juge sans la connaître, on la condamne, on la critique, on lui donne une majuscule qui l'élève au rang d'un dieu, mais on continue à croire que l'ère du digital est une sorte de truc de magicien. La plupart des intellectuels y voient la venue du dispositif, d'un pouvoir qui possède l'apparence incantatrice et menteuse du mobile, mais ils ne savent absolument rien des ondes hertziennes, de micro chips, de la structure de l'ordinateur. Et alors on fait vacance de la pensée, la pensée qui analyse et cherche à comprendre : on préfère haranguer le peuple contre le péril de la Technique, tout en favorisant le pouvoir dans sa démarche réifiante. Nous sommes fait de la même étoffe que la technique, parce que la technique c'est l'apax de notre culture, la même culture qui passe à travers nos gènes, qui nous rend des artéfacts, nous qui devrions être fiers d'être les fils de la roue et du feu, de la charrue comme de la guillotine quand elle nous a servi pour nous épurer et nous défendre de l'agresseur et de l'oppresseur, de nos vêtements comme des avions, des bâtiments comme des cavernes.

Et la France ne se trouvait sans doute pas dans une situation meilleure que l'Allemagne en 1940 : mais voilà, elle était la victime d'un agresseur, qui - et il faut le dire même si cela pèse en tant qu'italien - n'était pas seul pendant cette agression, parce qu'il trouvait dans une Italie complice et amorale un allié fort et maladroit qui venait 
décider des destins de la patrie de la Déclaration des droits de l'homme et du citoyen. Mais la France avait des intellectuels qui - et cela il faut le dire - comme ceux d'une Italie oubliée ou méconnue cherchaient l'action plutôt que le désespoir, croyaient dans l'homme plutôt que dans la race, avaient le courage de regarder dans les yeux la Gorgone et mouraient pour la liberté.

C'est donc seulement ainsi qu'on peut chercher à trouver ce qui à la fois lie et sépare Simondon et Anders : c'est la bataille éternelle entre ceux qui soulèvent les épaules et pleurent et ceux qui prennent en charge la misère et donnent une chance à leur peuple, en l'invitant à travailler, combattre et repenser le présent. Nous sommes en 1958, De Gaulle a institué la cinquième République, la France se soulève, Sartre prononce ses discours: c'est l'homme qu'on cherche, c'est la personne qu'il faut légitimer. Et l'homme, la personne coïncide avec ce qu'il fait, il n'y aurait jamais rien qui puisse le contraster. Simondon aussi l'avait vu et compris dès 1953 - voyez-vous, c'est la même date que le discours d'Heidegger - et il le disait, à claires lettres, dans un bref article, où il apparaît encore comme un des jeunes de l'Association, avec son prénom précédé par un J. plutôt qu'un G. :

Le chauvinisme de l'adolescent, l'impétuosité de son engagement parfois irréfléchi, ce besoin d'action, cette ardeur brûlante des désirs font de lui plutôt une force qui va qu'un être qui pense. Sa pensée est vie ou plutôt exigence de vie. Et pourtant il n'y a pas d'humanisme sans une certaine maturité, historique et individuelle. L'histoire des peuples comme la biographie des hommes montre que l'humanisme n'est pas primitif, et qu'il n'apparaît que lorsque l'être a su trouver son équilibre, sa mesure, comme était d'entéléchie défini par le plein accomplissement du mevtrion. Mais il n'y a pas non plus d'humanisme sans une certaine chaleur affective, sans une sorte d'enthousiasme et de montée de l'être. Le véritable humanisme sera donc un mixte de maturité et de jeunesse, si l'on définit par maturité le sens de la mesure interne et par jeunesse le sens de l'enthousiasme.

15 C'est donc ici qu'on peut trouver la première différence entre Anders et Simondon : ce sens $d u$ Tout qui transpire dans les mots du philosophe de MEOT, et l'air funèbre qui étouffe les pages d'Anders. Tous les deux avaient compris que la technique gagne une certaine autonomie, tous les deux l'avait regardée, mais seul Simondon l'avait vue vraiment, et pas parce qu'il possédait des yeux différents de ceux d'Anders, des yeux de l'âme, pour le dire avec Platon, mais parce que, tout au contraire, Simondon était le seul des deux qui avait les Yeux de la chair, parce que, toujours en le disant avec son œuvre de 1989, il se sentait frère des hommes, il leur reconnaissait le pouvoir de l'action.

On a dit que les deux philosophes avaient compris que la technique gagne une certaine autonomie : cela est absolument vrai. Mais si pour Anders cela signifie ce que Mary Shelley avait peint dans son Doctor Frankenstein - qu'Anders cite directement pour confirmer son point de vue - Simondon y voyait, vous le savez bien, une fête, semblable à celle célébrée par l'Encyclopédie de Diderot et d'Alembert. On pourrait donc résoudre le problème en affirmant, tout simplement, que c'est une question de sensibilités différentes, de subjectivités disparates mais pas destinées à une structuration? Je ne pense pas que la philosophie puisse être résolue dans une sphère subjective, même si ce sont toujours des sujets qui y contribuent. Je crois plutôt que la question se pose sur un plan de responsabilité philosophique: les journalistes, les éditorialistes peuvent quelquefois soulever des questions sans y donner une solution. La manie catastrophiste contemporaine est devenue un leitmotiv de la «société du spectacle » : ça sert - pour le dire avec Foucault - à mieux « surveiller et punir ». Mais les philosophes ont un devoir moral face à la société : c'est-à-dire celui d'expliquer et d'indiquer des voies. Simondon 
n'était pas l'optimiste exacerbé que quelqu'un nous a fait croire : dans ses articles sur la Psychosociologie de la technicité de 1960-1961 on le voit bien, en lisant ses analyses sur la manipulation appliquée par le marché à l'objet technique: faut-il se rappeler ces observations sur les voitures de luxe pour en avoir un témoignage? Mais Simondon vivait son époque et il sentait l'obligation morale de parler à ses contemporains pour répondre à la question qu'on a posée au début - «Que faire ?» - en leur donnant une solution humaine et finalement culturelle, s'il est vrai que l'homme est sa culture, et la «mémoire» de notre culture, comme dit Bergson, «nous suit toute entière ». La différence donc réside avant tout dans une façon de concevoir l'histoire, qui reste, pour Simondon, une affaire strictement humaine. Tout ce qui fait l'histoire, les sciences et les savoirs pour lesquels il faut trouver une méthode transductive de communication et pacification, en sont la trame. Mais comme les branches du même arbre - image que je prends à d'Alembert - ils appartiennent au même arbre qui est la faculté de l'intelligence, tendance humaine, qualité de l'homme. S'il faut donc relever des obstacles pendant le cours du développement historique, c'est toujours aux hommes qu'il faut parler et c'est l'homme qu'il faut présupposer. La mort de l'homme annoncée par Foucault n'était pas du même degré que celle bâtie par Anders : pour Foucault c'est l'idée d'homme qui meurt, pour Anders ce sont les hommes particuliers qui disparaissent du scénario de l'histoire. Et la responsabilité en incombe à la Technique, détentrice d'une morale bien différente de celle de ses créateurs... voilà donc le problème qui s'approche : qu'est-ce que la technique pour Anders et pour Simondon? Ou bien, est-ce qu'il existe une technique pour Simondon? Anders, on l'a déjà vu, avait fait de la technique le nouveau sujet de l'histoire: faut-il donc la penser comme douée d'un corps, d'un esprit, d'une volonté ? La question peut sembler bizarre, mais ce n'est pas comme ça, parce que pour Anders, la Technique possède un corps unique, mieux elle est substantielle, donc elle est cette chose qui ne nécessite rien d'autre pour exister que soimême, et sa structure physiologique - peut-on dire avec Serge Latouche - c'est la mégamachine. Si vous vous demandez - comme je l'ai fait - où peut-on rencontrer ce corps, vous devriez répondre avec les néo-positivistes : la question est mal posée, même dieu on ne l'a jamais rencontré et pourtant il y a quelqu'un qui prétend le représenter sur terre. Au contraire, Simondon ne pose jamais la question d'une existence unique de la technique, il préfère analyser les techniques, qui sont les seules dont on puisse témoigner et que nous avons le devoir de connaître. Cela signifie avant tout que Simondon - même indirectement - partage une observation faite par Bachelard à l'égard des sciences et de la réalité : «le simple - disait Bachelard dans La naissance de l'esprit scientifique - n'existe pas dans la réalité. C'est plutôt la science qui simplifie avec ses lois. » Et Simondon reste en contact, comme avait fait l'épistémologie historique de Bachelard et de Canguilhem, avec cette réalité : c'est pour cela que ses généralisations sont toujours des généralisations concrètes, comme le démontre sa profondeur d'analyse des objets techniques, qui ne sont pas des choses et des instruments, comme aurait dit Heidegger (cela Simondon le savait et le critiquait toujours dans ses articles de 1960 et 1961). Voilà donc qu'on voit apparaître un autre paradoxe dans la réflexion d'Anders : sa philosophie était, ou bien devrait être, une critique des conséquences des sciences exactes ou plus encore des conséquences techniques et technologiques (même si pour Anders il n'y a aucune différence entre les deux secteurs) donc des disciplines qui se fondent sur un système de règles fait de généralisations, même probabilitaires comme l'a montré la physique quantique. Et tout au contraire c'est Anders qui généralise, sans vraiment connaître ce dont il parle, en se limitant à un examen, qu'on pourrait peut- 
être définir comme sociologique, du phénomène technique, mais qui reste à la surface du problème. Simondon recueille ce problème et il est capable de lui donner un nom : nous sommes dans un secteur qui a soulevé des critiques à l'égard de Simondon, surtout par les marxistes, mais qui à mon avis révèle une puissance innovatrice : l'aliénation - dit Simondon - n'est pas une condition réservée aux ouvriers. Elle est plutôt la condition dans laquelle se trouvent - paradoxalement - les machines dans l'époque des machines. Elles sont méconnues, oubliées comme les mains qui servent une société mais qui n'obtiennent pas la juste reconnaissance. À mon avis, c'est la réflexion d'Anders qui fait contrepoids à cette thèse : sa rage contre les avions de l'ennemi, la constatation des ruines, l'obligent à condamner le réseau technique qu'il y aurait aux épaules de la Guerre : la Machine totale. Et maintenant, il faudrait se demander si Anders connaissait les machines dont il parlait. Sa vie l'a vu ouvrier à la chaîne de montage, chargé de la haine que ce travail peut provoquer et la chaîne l'a transformé en luddiste, privé de la force de se révolter, prophète d'une eschatologie qui lui dérobe la seule façon de réagir à l'absurde - pour le dire avec Camus-, c'est-à-dire «la révolte». Simondon, naturellement, n'imaginait pas un syndicat des machines quand il parlait d'aliénation, mais il parle aux hommes, ceux que Anders continuait à entendre comme des mémoires $d u$ sou-sol. L'appel de Simondon correspond, donc, à une prise de conscience, la plus digne des activités que les philosophes peuvent exercer : c'est la meilleure des nékuia qu'on peut pratiquer à la mémoire de Socrate, l'homme en lequel Merleau-Ponty, dans son Éloge de la philosophie, avait reconnu le modèle le plus emblématique du philosophe.

Je ne connais pas exactement la situation de la philosophie en France aujourd'hui, et surtout je ne connais pas son rapport aux techniques, sauf la position de Bruno Latour à cet égard. Ce dont je peux témoigner, c'est naturellement de ce que les philosophes italiens, pour la plus part d'entre eux, pensent à l'égard de cet argument. Nous avons assisté, dans les dernières vingt années, à une véritable diffusion des suggestions heideggériennes, et cela a conduit quelqu'un a partager aussi la perspective d'Anders, en cherchant à retrouver une solution à la question de la technique en proposant une récupération des mythes grecs, tout en oubliant que même l'usage des mots grecs, aujourd'hui, ne peut pas nous offrir un véritable contact avec les Grecs, puisque comme on devrait le savoir - et comme Vernant nous l'a plusieurs fois rappelé - parler des Grecs cela pourrait seulement vouloir dire imaginer ce qu'ils ont cherché de dire : la récupération, donc, c'est un effort pénible, une passion inutile. D'autre part, cette façon d'approcher le présent n'est pas une modalité consciente et active, comme il me semble au contraire que la philosophie devrait faire. C'est pour cela que je crois qu'il faudrait encore une fois en appeler aux mots de Simondon : l'aliénation technique qu'on a vu s'exprimer aussi par la bouche d'un des philosophes allemands plus à la page comme Anders, ne correspond pas seulement à une méconnaissance des machines par les individus singuliers. Il est plutôt la coutume de toute une culture qui renonce à la compréhension des phénomènes actuels en songeant à un âge d'or ou plus encore en se désespérant à cause d'un présent qui ne semble préparer aucun futur. C'est ainsi qu'on peut comprendre les mots qui ouvrent le texte que Simondon a consacré à la technique : quand il dit que «la culture s'est constituée en système de défense contre les techniques " cela pourrait nous sembler absurde si on regarde la diffusion des techniques contemporaines dans le monde d'aujourd'hui. Mais c'est là qu'il me semble le moment de rappeler un aphorisme d'Oscar Wilde selon lequel «ce que nous ne voyons pas correspond exactement à ce que nous avons sous les yeux ». Qu'est-ce que ça veut dire? Vous le voyez, nous sommes dans l'ère d'Internet et on prépare aussi un 
WEB 2 qui servira pour transposer une quantité majeure des donnés, et toutefois, qu'est-ce que c'est vraiment qu'Internet, ou bien, comment considère-t-on aujourd'hui Internet? On pense à Internet seulement comme à un instrument et comme un instrument dangereux : je ne sais pas si vous avez entendu l'intention de notre ministre de la Justice, qui maintenant a été la cause de la chute du gouvernement pour le scandale qu'il a provoqué avec la magistrature, qui pensait à une commission de contrôle pour Google. Cela porte un seul nom: censure. Je ne veux pas dire que l'éthique ne doit pas interagir avec la science et la technique, mais je suis vraiment préoccupé par le fait que quand on parle de technique il faut toujours qu'on nous rappelle seulement les dommages qu'elle peut causer. C'est un vieux discours, un discours qui, pensez-vous, était central aussi dans l'Éthique d'Abélard, qui présentait l'oreiller soit comme un confort soit comme une arme pour étouffer. Mais le problème, et Simondon l'avait compris, c'est le fait qu'on pense à la science et plus encore à la technique à la manière d'Anders : comme un moyen qui se substitue aux buts et qui habite exclusivement la sphère réservée aux esclaves. C'est pour cela que le premier chapitre de la thèse de doctorat de Simondon n'est pas seulement un examen de l'individuation de l'inorganique, mais aussi et surtout une apologie de la technique comme expression d'un faire et d'un savoir : la brique d'argile ne serait pas ce qu'elle est sans l'énergie de l'ouvrier, sans sa connaissance de la matière, de ses propriétés colloïdales, de sa résistance. Et encore, voilà un exemple qui permet de comparer l'ouverture de la pensée simondonienne à un certain snobisme heideggérien : pour Heidegger, on l'a déjà dit, le temps des bûcherons est passé, maintenant c'est l'ère de l'excavateur ; au contraire, Simondon y voit le temps d'une nouvelle compétence, d'une nouvelle sagesse : celle de la scie à ruban, de la clavette etc. En suivant sa voie, nous nous trouvons à repenser les machines en termes d'objets techniques plutôt que d'instruments, et cela veut dire comme des synergies de structures et d'opérations, de théories aiguës et d'applications que j'aimerais définir comme démocratiques. Je suis sûr que vous vous rappelez les mots que Canguilhem avait dédiés au phénomène des cultures génétiquement modifiées. Il ne faut pas s'enthousiasmer trop : nous ne serions pas de véritables humanistes selon l'expression de Simondon, mais il faut toutefois reconnaître avant tout les emplois positifs que la technique, ou bien les techniques pourraient avoir en vue d'une progressive libération de la société. Internet cache sans doute des trucs et obstacles, mais l'heuristique de la peur - comme la voulait Jonas - ne peut jamais représenter une solution. Il y a un autre aspect qu'il faut se rappeler: connaître quelqu'un, ou bien quelque chose, c'est connaître et respecter son histoire. Anders avait tué l'histoire, mais il démontre aussi que ce dont il parle - c'est-à-dire la Technique - ne semble pas en avoir une. Au contraire, voilà que Simondon nous invite à faire l'histoire des objets : c'est un défi stimulant, mais qui aujourd'hui ne semblerait pas avoir rencontré de véritables succès. Vous le voyez quotidiae: tous les jeunes utilisent Internet, mais quand on parle d'Arpanet ou bien du CERN on a l'impression d'être regardé comme un alien. D'où vient-il cet esprit de finesse de Simondon pour la technique et plus encore cette exigence de connaissance de ses phénomènes? Vincent Bontems et Jean-Hugues Bathélémy l'ont dit bien et avec force : c'est une origine digne et libératrice qui semble récapituler pas seulement l'histoire de la France mais celle de toute l'Europe moderne et contemporaine, c'est l'esprit encyclopédique des illuministes, un esprit méconnu par Anders et par ceux qui y voyaient le symptôme d'une catastrophe future. La mission de l'intellectuel que nous lègue Gilbert Simondon, c'est un effort qui nécessite surveillance et fraîcheur de pensée et l'envie de révolte 
face au statu quo de la culture, d'une culture qui aujourd'hui semble préférer la prostitution du spectacle des média à tout encyclopédisme. Toutefois, il faut croire à ce défi, et alors laissez-moi terminer avec une invitation illuministe qui reste une invitation pour tous les temps, une invitation qui nous éperonne en criant : «Citoyens, allez en avant ! La foi vous viendra!»

\section{BIBLIOGRAPHIE}

Anders Günther, Die Antiquiertheit des Menschen, C.H.Beck, 2002.

Bloch Marc, Marc Bloch. Apologie pour l'histoire, Paris, A. Colin, 1949.

Heidegger Martin, Sein und Zeit, Berlin, Akademie Verlag, 2001.

Spengler Oswald, Le déclin de l'Occident : esquisse d'une morphologie de l'histoire universelle, Paris, Gallimard, 1931-1935.

Spengler Oswald, L'homme et la technique [1931], Anatole A. Petrowsky (trad.), Paris, Gallimard, 1958.

INDEX

Personnes citées : Anders (Günther), Simondon (Gilbert), Heidegger (Martin)

Mots-clés : technique

\section{AUTEUR \\ GIOVANNI CARROZZINI}

Professeur de philosophie en Italie, il a publié un livre sur Simondon : Gilbert Simondon : per un'assiomatica dei saperi. Dall "ontologia dell'idividuo" alla filosofia della tecnologia, Manni, 2006. 